\title{
Stellar feedback and triggered star formation
}

\section{Jan Palouš, ${ }^{1}$ Richard Wünsch, ${ }^{1}$ James E. Dale, ${ }^{1}$ Vojtěch Sidorin ${ }^{1}$ and Anthony Whitworth ${ }^{2}$}

\author{
${ }^{1}$ Astronomical Institute, Academy of Sciences of the Czech Republic, \\ Boční 1401, CZ-14061, Prague 4, Czech Republic \\ email: palous@ig.cas.cz \\ ${ }^{2}$ School of Physics and Astronomy, Cardiff University, Queens Buildings, \\ The Parade, Cardiff CF24 3AA, UK \\ email: Anthony. Whitworth@astro.cf.ac.uk
}

\begin{abstract}
Young, massive stars influence their ambient medium through winds and radiation. The outcome of this feedback depends on the number of massive stars in a star cluster and on the density of the ambient medium. This contribution is based on a comparison of observations to the results of numerical simulations. We discuss the gravitational fragmentation of feedbackdriven shells expanding from young stellar clusters. The thin-shell approximation is compared to 3D hydrodynamical simulations with smoothed-particle hydrodynamics and adaptive-mesh refinement codes. We explore the influence of external pressure and propose a thick-shell dispersion relation, where the pressure of the external medium is included. The mass spectrum of the shell fragments is constructed and we speculate about the origin of the deficit of low-mass objects.
\end{abstract}

Keywords. stars: formation, ISM: structure, galaxies: star clusters

\section{Introduction}

In the Milky Way (McClure-Griffiths et al. 2002; Ehlerová \& Palouš 2005) and in nearby galaxies within $15 \mathrm{Mpc}$ (Walter et al. 2008), Hi observations reveal a plethora of structures, some of which are shells with sizes of 10-1000 pc and expansion velocities of 5-40 $\mathrm{km} \mathrm{s}^{-1}$. Using the Spitzer GLIMPSE survey, Churchwell et al. (2006, 2007) found many smaller $(0.1-10 \mathrm{pc})$ and younger structures $(<1 \mathrm{Myr})$, frequently surrounding young stellar clusters. In some cases (e.g., RCW 79 Deharveng et al. 2007), the shells are fragmented and some of the fragments harbor young stellar clusters visible in the near-infrared (near-IR).

In a search for Hi counterparts of the IR structures revealed by the GLIMPSE survey, Sidorin (2008) identified several regions with shapes showing striking similarities. The high-angular-resolution Hi data collected with the Arecibo telescope as part of the I-GALFA project have shown an even better correspondence (see Figure 1). The identification of IR structures in HI observations provides radial velocities, which may be used to estimate distances, sizes and also thicknesses of the shell-like structures. For N107, we derive a distance of $4.7 \mathrm{kpc}$, a size of $15.5 \mathrm{pc}$ and a thickness of $3.1 \mathrm{pc}$. Several young stellar objects are connected to this bubble.

We conclude that there is a growing collection of expanding shells seen in HI, and at optical and IR wavelengths, with evidence of fragmentation and triggered formation of new stellar clusters along their edges. 

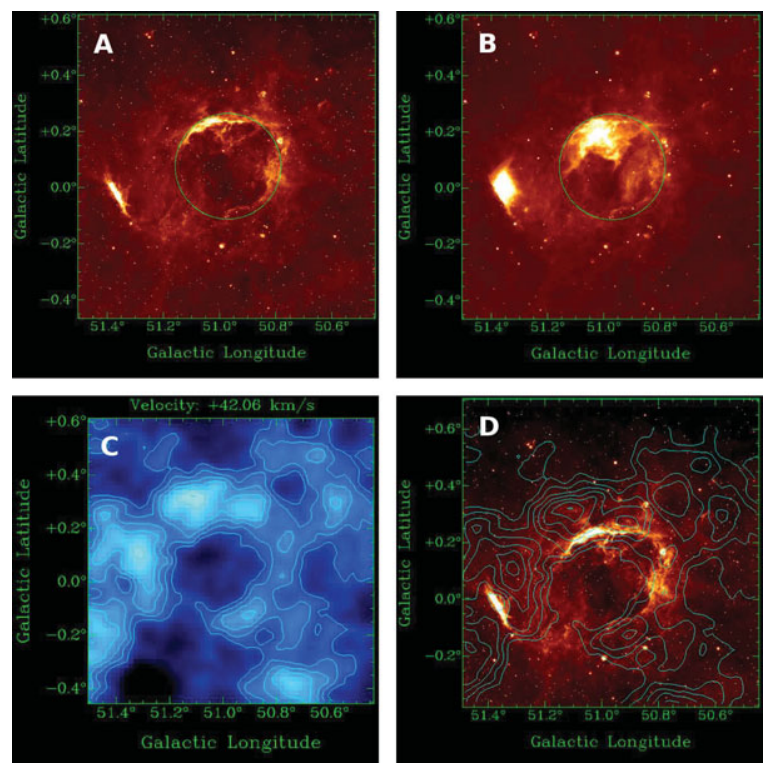

Figure 1. Bubble N107 (green circle) of Churchwell et al. (2006) as seen in Hi. (a) Spitzer/IRAC, $8 \mu \mathrm{m}$. (b) Spitzer/MIPS, $24 \mu \mathrm{m}$. (c) Arecibo, I-GALFA, Hi line $(21 \mathrm{~cm})$, radial velocity $42 \mathrm{~km} \mathrm{~s}^{-1}$ : similar to the radial velocities of the HiI regions associated with the bubble. (d) Background: Spitzer/IRAC, $8 \mu \mathrm{m}$; contours: Arecibo, I-GALFA, HI.

\section{SPH and AMR simulations: pressure confinement}

The investigation of gravitational fragmentation of expanding shells using analytical and numerical analysis by Dale et al. (2009a) reveals the constraints of the thin-shell approximation as developed by Vishniac (1983), Elmegreen (1994), Whitworth et al. (1994) and Wünsch \& Palouš (2001). With two different numerical approaches, Eulerian stationary adaptive-mesh refinement (AMR) and Lagrangian smoothed particle hydrodynamics (SPH), several 3D numerical experiments have been performed. The setup of these experiments assumes momentum-driven shells expanding into a very-low-density environment with low or high thermal pressure. We do not study the influence of ram pressure. Its effect can differ from that of thermal pressure and it will introduce other instabilities, which we try to avoid here.

The results of the AMR and SPH codes and the analytical prediction of the linear thin-shell analysis are compared. We observe the expanding-shell fragmentation process and arrive at the following conclusions (Dale et al. 2009a):

- the AMR and SPH results agree extremely well;

- the thermal pressure of the external environment strongly affects the fragmentation process;

- expansion into a low-pressure environment forms high-mass fragments only;

- confinement of the shell with high external pressure leads to the formation of fragments with a mass spectrum predicted by the thin-shell analysis.

\section{Thick-shell dispersion relation}

In an attempt to disentangle the role of external pressure, we introduce a shell in hydrostatic equilibrium between internal pressure and its own gravity, surrounded by an environment imparting external pressure. Such a thick shell, with a given surface density, 


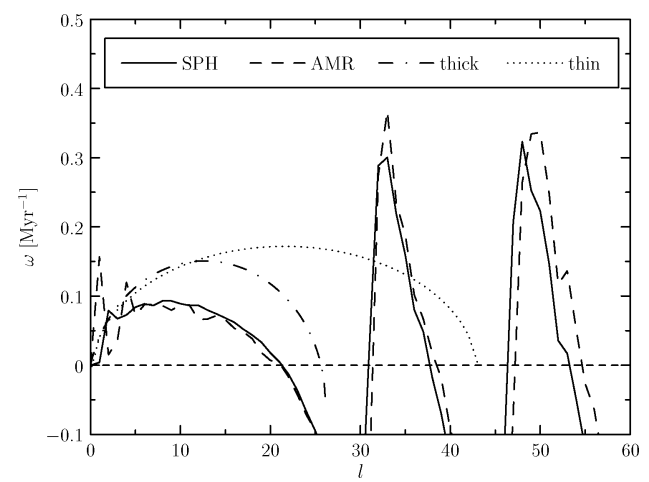

Figure 2. Dispersion relation of a shell expanding into a low-pressure environment. The thickand thin-shell dispersion relations are compared to dispersion relations derived from AMR and SPH numerical experiments.

thickness and internal sound speed is subjected to perturbations and we derive the thickshell dispersion relation. The growth rate of the fragments is not only a function of the shell's expansion velocity, its radius, surface density and internal sound speed, like in the thin-shell case. In the thick-shell dispersion relation, the external pressure is also included. The detailed derivation of this relation is given in Wünsch et al. (in prep.).

In Figure 2 we show a comparison of the numerical results from our AMR and SPH codes for thin-shell and thick-shell dispersion relations for a shell expanding into a lowpressure environment. The thick-shell dispersion relation shows much better agreement with the results of the numerical simulations. It also predicts a deficit of low-mass fragments.

The thick-shell dispersion relation leads to the following conclusions:

- for low external pressure, low-mass fragments are underabundant;

- there is a critical value of external pressure, which is a function of the shell's surface density, when the shell-fragmentation process is in agreement with the thin-shell prediction;

- for a given shell surface density, at the critical value of the external pressure, the thickness of the shell is greatest, compared to other values of the external pressure;

- for high external pressure, low-mass fragments are overabundant.

\section{Mass spectrum of fragments}

Palouš (2007) used linear thin-shell analysis to derive a mass spectrum following a Salpeter-like power law at the high-mass end and a turnover at low masses. In our numerical experiments with the AMR and SPH codes, we also analyze the mass spectrum of the fragments. We follow the fragmentation process using the potential-based clumpfinding algorithm of Smith et al. (2008).

The different stages of shell evolution are represented in Figure 3. In the early stages, the mass spectrum shows a high-mass end decline similar to the Salpeter slope. However, later on the mass spectrum is top heavy, with a strong deficit of low-mass fragments. The mass evolution of individual fragments in the numerical simulations shows how the early fragments accrete mass, leaving increasingly lower-density environments for those fragments that form later. A more detailed analysis of the mass-accretion process onto the fragments forming from an expanding shell is the subject of a forthcoming article (Dale et al., in prep.). 

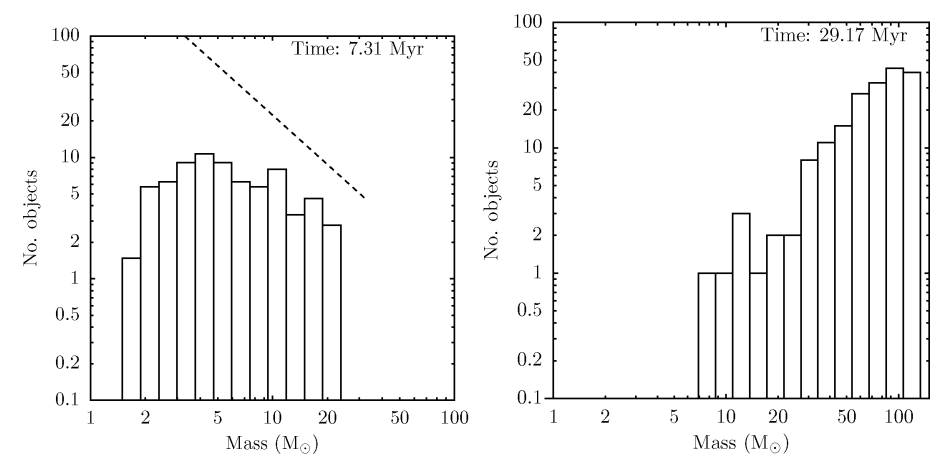

Figure 3. Time evolution of the fragment mass spectrum. (left) Early stage. The dashed line represents the Salpeter-like slope with a spectral index of -2.35 . (right) Late stage.

The preliminary results of our clump-evolution analysis show that:

- the main fraction of the shell mass is accreted by the first objects, while the latecomers are starved since not much gas is left by the objects that formed earlier;

- shells expanding into a low-density, high-pressure environment develop top-heavy fragment mass functions;

- for mass replenishment from a dense shell environment (e.g., if the shell expands into a dense molecular cloud), more low-mass objects may be formed.

\section{Conclusions}

We conclude from our analysis of the fragmentation of expanding shells that:

(a) the pressure in the shell environment plays an important role and the thin-shell analysis is applicable only when the external pressure has a certain predicted value;

(b) linear analysis on the basis of the dispersion relations for thin or thick shells describes the early stages of the fragmentation process only. Subsequently, nonlinear processes dominate mass accretion;

(c) the accretion process makes the fragment mass spectrum top heavy. Low-mass objects may form through accumulation of new mass by the shell from its environment.

\section{Acknowledgements}

JED acknowledges support by the EU FP6 'Constellation' project (MRTN-CT-2006035890). RW, VS and JP acknowledge support from the Institutional Research Plan AV0Z10030501 of the Academy of Sciences of the Czech Republic and project LC06014 Centre for Theoretical Astrophysics of the Ministry of Education, Youth and Sports of the Czech Republic.

\section{References}

Churchwell, E., et al. 2006, ApJ, 649, 759

Dale, J. E., Wünsch, R., Whitworth, A., \& Palouš, J. 2009, MNRAS, 398, 1537

Deharveng, L., Zavagno, A., Lefloch, B., Caplan, J., \& Pomarès 2007, Proc. IAU Symp. No. 237, p. 212

Ehlerová, S \& Palouš, J. 2005, A\&A, 437, 101

Elmegreen, B. G. 1994, ApJ, 427, 384

McClure-Griffiths, N. M., Dickey, J. M., Gaensler, B. M., \& Green, A. J. 2002, ApJ, 578, 176

Palouš, J. 2007, Proc. IAU Symp. No. 237, p. 114 
Sidorin, V. 2008, IR, optical and X-ray counterparts of HI shells in the Milky Way, MSc Thesis, Charles University, Prague

Smith, R., Clark, P., \& Bonnell, I. 2008, MNRAS, 391, 1091

Vishniac, E. T. 1983, ApJ, 274, 152

Walter, F., Brinks, E., de Blok, W. J. G., Bigiel, F., Kennicutt Jr., R. C., Thornley, M. D., \& Leroy, A. 2008, AJ, 136, 2563

Whitworth, A. P., Bhattal, A. S., Chapman, S. J., Disney, M. J., \& Turner, J. A. 1994, MNRAS, 268, 291

Wünsch, R. \& Palouš, 2001, A\&̛A, 374, 746 